\title{
Changing the Brain, Changing the Society: Clinical and Ethical Implications of Neuromodulation Techniques in Neurology and Psychiatry
}

\author{
Patrik Vuilleumier • David Sander • \\ Bernard Baertschi
}

Received: 15 October 2013/Accepted: 16 October 2013/Published online: 25 October 2013

(C) Springer Science+Business Media New York 2013

\begin{abstract}
New neurotechnologies for modulating brain functions are becoming increasingly powerful and applicable to diverse diseases, but raise new questions for clinicians, ethicists, policy makers, and laymen. This issue of Brain Topography gathers several papers exploring the clinical and ethical implications of several invasive and noninvasive neuromodulation approaches. The opportunities and challenges associated with the emergence of neuro-engineering solutions to brain research and brain repair require a close dialogue between multiple disciplines, as well as open discussion with the general public.
\end{abstract}

Keywords Neuromodulation · Deep brain stimulation · DBS · TMS · Neurofeedback ·

This is one of several papers published together in Brain Topography in the "Special Issue: Clinical and Ethical Implications of Neuromodulation Techniques".

P. Vuilleumier $(\bowtie)$

Department of Neuroscience, University Medical Center, Geneva, Switzerland

e-mail: patrik.vuilleumier@unige.ch

P. Vuilleumier · D. Sander

Center for Neuroscience, University of Geneva, Geneva, Switzerland

P. Vuilleumier - D. Sander

Swiss Center for Affective Sciences, University of Geneva,

Geneva, Switzerland

D. Sander

Department of Psychology and Education Sciences, University of Geneva, Geneva, Switzerland

B. Baertschi

Biomedical Ethics Institute, University of Geneva, Geneva, Switzerland
Neuroimaging $\cdot$ Neuroprosthetics $\cdot$ Brain machine interface

In the past two decades, tremendous progress has been made in brain sciences. We are just beginning to envision their enormous impact for many clinical conditions as well as their consequences for more global societal issues. It is likely that under the influence of new neuroscience techniques and knowledge, the clinical practice of neurology and psychiatry is about to witness major changes in diagnosis and treatment which will not only revolutionize medicine, but possibly also change our vision of human nature.

Modern brain research is now capable of elucidating the detailed neuronal circuitry involved in various behaviors and cognitive functions. It becomes possible to link complex mental states or phenomena to their underlying biological processes at various levels of analysis. This understanding is growing at a rapid pace and ranges from large-scale corticosubcortical networks through to synaptic, cellular, and subcellular systems pertaining to molecular, genetic, and even epigenetic mechanisms. Moreover, the realm of mental and brain processes that are now amenable to mechanistic biological explanations is spreading to encompass some of the most private and apparently most intangible facets of the human mind-such as emotions, memory, or morality (Baertschi 2014; Glannon 2014).

This progress in our knowledge of the brain has been made possible thanks to the advent of several breakthroughs in technologies and experimental tools that did not exist 15 or 20 years ago. These include (among others) neuroimaging methods, transcranial magnetic stimulation, and deep brain electrodes in humans, but also patch-clamp, knock-out, viral transfection, or optogenetics in animals. 
Furthermore, for the first time in neuroscience history, some of these techniques might potentially be applied in similar ways to both humans and animals, thus allowing much more direct and faster integration of knowledge gained at different scales of neuronal functions. The convergence between neurobiology, neuroimaging, and neuroengineering opens unprecedented perspectives for translational research.

But even more importantly, these technological advances open many new perspectives that will now allow researchers and clinicians not only to measure brain activity during complex mental states or cognitive tasks, but also to interfere or modulate neural processes in a way such as to influence mental experience and behaviors of patients. Some technologies with this potential for interventions are already widely available, including invasive methods such as transcranial or intracranial stimulation (Schlaepfer and Bewernick 2014; Cabrera et al. 2014), but also novel use of non-invasive imaging methods (such as functional magnetic resonance imaging or electroencephalography) for real-time control of brain activity and neurofeedback (Birbaumer et al. 2014; Gevensleben et al. 2014). In addition, progress at the molecular and genetic levels has induced (and will further promote) the development of new drugs that can change brain functions and possibly alter its synaptic architecture in a long-lasting (perhaps permanent) manner (Fins and Shapiro 2014). Fascinating therapeutic applications can now be envisioned in neurology as well as psychiatry for each of these new tools, plus their potential combination with each other or with more traditional approaches (including purely behavioral training procedures or even psychotherapy). It is for example becoming plausible to imagine the potential therapeutic benefits of protocols that would complement neurological rehabilitation procedures after brain injury with direct brain stimulation and specific drug regimens in order to modulate synaptic plasticity, or procedures that would enhance psychotherapeutic treatment with concomitant neurofeedback based on regional brain activity and/or with drugs modulating genetic expression in select neuronal populations.

Such advances not only offer exciting prospects that illustrate the immense accomplishment of science, medicine, and technology, but also more generally highlight the captivating deepening of our knowledge about brain, mind, and human nature. These advances also raise many new questions and fears about the safety, limits, and ethical framework that should define their applications and development. The possibility to influence brain function in a powerful manner for psychiatric diseases, for example, leads to delicate and unprecedented questions about privacy, individuality, personal identity, or consent (Glannon 2014). In addition, the fact that we have progressively gained a vast knowledge gained about the mechanisms of these techniques and the neural substrates of mental processes makes their effects on the mind even more salient and graspable by the general public, which may further alarm about their potential or perceived danger. Hence, focal and reversible brain stimulation is still seen as more invasive than the administration of psychotropic drugs, which are often well accepted by the public, even though the latter may produce much more widespread effects on the both brain and body, and occasionally also affect neural circuitry in an irreversible manner. In addition, the emergence of new possibilities to influence brain function with refined technologies is likely to revive some passionate debates and dark pages of the past history of neuropsychiatry, in relation to lobotomy and electroconvulsive therapy. The well-known abuse and unfortunate haste in their applications in the twentieth century has generated not only caution but also negative biases against similar interventions, which may ultimately be counterproductive and detrimental to real therapeutic benefits for some patients. It is therefore important that emerging new technologies do not commit a similar fault, but also that they do not suffer from unjustified generalization of fears.

Indeed, besides the fascination, mystery, and worry of unwise applications of new intervention techniques to regulate brain function, there are also great hopes that at least some of these approaches will improve mental or neurological diseases and thus alleviate major causes of human suffering. To guarantee an optimal development of these techniques by scientists and their acceptance by patients and their families, it is of paramount importance to clarify their effects and limitations and to discuss them in comparison with other current therapeutic means.

These questions were at the heart of a recent symposium organized at the Brocher Foundation in Geneva, an institution devoted to promote meetings and reflections around the ethical and societal implications of modern medicine (http://www.brocher.ch). During 2 days in June 2012, this symposium brought together several neuroscientists working at the frontiers of new technological developments for therapeutic purposes, as well as philosophers and ethicists, who engaged in lively discussions with each other and with a public audience composed of clinicians, engineers, policy makers, and laymen. This symposium illustrated the breadth of current and future technological opportunities, both for the treatment of neuropsychiatric diseases and for the enhancement of normal brain functions. Several speakers who participated to the symposium have subsequently decided to write review or opinion papers which are now gathered in a special section of this issue of Brain Topography.

In line with lectures and discussions that took place during the symposium, the papers gathered in this issue 
critically discuss the benefits and limitations of these techniques at the present time. However, the global overview offered by these authors clearly makes the case that the future will bring powerful intervention methods that will not only concern motor control in Parkinson's disease, stroke, or tetraplegia (Birbaumer et al. 2014; Cabrera et al. 2014), but also increasingly target higher-level mental functions such as mood and anxiety regulation (Schlaepfer and Bewernick 2014), as well as memory, attention, impulsivity (Gevensleben et al. 2014), or even social behavior and morality (Baertschi 2014). In virtually each of these domains, neural intervention techniques are currently being explored through various approaches including deep brain stimulation, TMS, neurofeedback, pharmacology, and even innovative neuroprosthetics devices.

Importantly, in all cases, efficient interventions will require a precise understanding of brain circuits and sophisticated means to decode neural activity at both the local and large-scale levels. Hence, this endeavor will be intimately connected with the development and validation of neuroimaging techniques by authors and readers of Brain Topography. Moreover, there is not only increasing refinement in brain imaging methods and signal processing tools in neuroscience, but also growing interest for application and cooperation in industry, which will undoubtedly contribute to rapid expansion of a new field of neurobioengineering. Time is therefore ripe for thinking about the objectives, limitations, risks, and priorities for new neurotechnologies (Fins and Shapiro 2014; Glannon 2014). Research published in Brain Topography will continue to steer these developments by providing innovative tools to assess brain function, but also actively contribute to establish the best usage of such tools and to educate the public and policy-makers alike, with the ultimate goal to help patients and improve society in accordance with rigorous ethical guidance.

\section{References}

Baertschi B (2014) Neuromodulation in the service of moral enhancement. Brain Topogr. doi:10.1007/s10548-012-0273-7

Birbaumer N, Gallegos-Ayala G, Wildgruber M, Silvoni S, Soekadar SR (2014) Direct brain control and communication in paralysis. Brain Topogr. doi:10.1007/s10548-013-0282-1

Cabrera LY, Evans EL, Hamilton RH (2014) Ethics of the electrified mind: defining issues and perspectives on the principled use of brain stimulation in medical research and clinical care. Brain Topogr. doi:10.1007/s10548-013-0296-8

Fins JJ, Shapiro ZE (2014) Deep brain stimulation, brain maps and personalized medicine: lessons from the human genome project. Brain Topogr. doi:10.1007/s10548-013-0297-7

Gevensleben H, Kleemeyer M, Rothenberger LG, Studer P, FlaigRöhr A, Moll GH, Rothenberger A, Heinrich H (2014) Neurofeedback in ADHD: further pieces of the puzzle. Brain Topogr. doi:10.1007/s10548-013-0285-y

Glannon W (2014) Neuromodulation, agency and autonomy. Brain Topogr. doi:10.1007/s10548-012-0269-3

Schlaepfer TE, Bewernick BH (2014) Neuromodulation for treatment resistant depression: state of the art and recommendations for clinical and scientific conduct. Brain Topogr. doi:10.1007/ s10548-013-0315-9 